\title{
Microwave spectrum and structure of the dimethylamine dimer: Evidence for a cyclic structure
}

\author{
Michael J. Tubergen and Robert L. Kuczkowski \\ The Department of Chemistry. The University of Michigan, Ann Arbor, Michigan 48109-1055
}

(Received 13 September 1993; acceptcd 17 November 1993)

\begin{abstract}
Rotational spectra have been measured for six isotopomers of the dimethylamine dimer using a Fourier-transform microwave spectrometer. No tunneling splittings were observed for either the $a$ - or $c$-type rotational transitions and the spectra could be fit to a rigid rotor Hamiltonian including centrifugal distortion terms. Possible dimer structures are discussed and compared to the experimentally determined moments of inertia, dipole moments, and nuclear quadrupole coupling constants. A cyclic structure with $C_{s}$ symmetry was found to best reproduce the inertial data. The structure of the dimethylamine dimer is compared to the theoretical and experimental structures for the ammonia dimer.
\end{abstract}

\section{INTRODUCTION}

The recent sensitivity gains of high resolution spectrometers has enabled the detailed study of weakly bound complexes. In particular, high resolution spectroscopy has been applied to complexes which contain intermolecular hydrogen bonds because hydrogen bonding is implicated in the structures and stabilities of very complicated systems. Experimentally determined structures are often compared to theoretically predicted structures and the agreement between experiment and theory has generally been good.

The ammonia dimer, however, has proven to be a particularly challenging system for theorists and experimentalists alike. The first high resolution microwave experiments ${ }^{1}$ on the ammonia dimer utilized molecular beam electric resonance and Fourier-transform microwave spectrometers to resolve the nuclear quadrupole hyperfine components and measure the Stark shifts of a pair of tunneling doublets associated with the $J=0-1$ and $J=1-2, K=0$ transitions. The doublets were assigned to an interchange tunneling motion which exchanges the roles of the two monomers within the dimer. Structural information about the ammonia dimer was deduced from the value of ( $B$ $+C) / 2$ and by relating the measured values of the dipole moment and quadrupole coupling constants to the monomer values. This method determined the center-of-mass distance and the absolute values of the tilt angles $\left(\theta_{1}\right.$ and $\theta_{2}$ ) of the monomer symmetry axes with respect to the line joining the monomer centers of mass. The values of $\theta_{1}$ and $\theta_{2}$ were found to be inconsistent with dimer structures containing linear $\mathrm{N}-\mathrm{H}-\mathrm{N}$ hydrogen bonds and instead suggested that the dimer exists in a cyclic form. Finally, the structural data were found to be consistent among the isotopes studied, suggesting to the authors that the data are not contaminated by averaging over large amplitude vibrational motions. The initial spectroscopic data on the ammonia dimer, in combination with spectroscopic data from other ammonia complexes, were sufficient to raise doubts about the ability of ammonia to act as a hydrogen bond donor. $^{2}$

Extensive theoretical work has been done on the ammonia dimer and a thorough review is beyond the scope of this introduction. Two early theoretical treatments supported cyclic equilibrium structures for the ammonia dimer, ${ }^{3}$ but these calculations did not locate the true minimum of the potential energy surface or used basis sets that were found to be too small. A very recent, high level calculation employing bond functions finds a cyclic, $C_{2 h}$ dimer structure to be more stable than a linearly hydrogen bonded $C_{s}$ structure. ${ }^{4}$ However, most other optimized structures from $a b$ initio and semiempirical calculations contain linear hydrogen bonds. ${ }^{5}$ Because the theoretical work has also consistently shown that the potential energy surface is very flat, it has been suggested that the spectroscopically determined dipole moment and quadrupole coupling constants contain large vibrational averaging effects and that the structure derived from them may not be descriptive of the structure at the minimum of the potential energy surface. ${ }^{5(c), 5(d), 6}$

A vast number of vibration-rotation-tunneling (VRT) transitions have recently been measured for the ammonia dimer. ${ }^{7,8}$ From analysis of the VRT spectrum it was determined that the molecular symmetry group for the ammonia dimer is $G_{144} \cdot{ }^{8}$ Three different tunneling motions were found to be feasible for the dimer-internal rotation about one or both of the monomer $C_{3}$ axes, monomer inversion tunneling, and interchange tunneling. It was also found that the interchange tunneling frequency is very large (16.1 and $19.3 \mathrm{~cm}^{-1}$ for the $A+A$ and $E+E$ internal rotation states) and that the tunneling splitting observed in the microwave spectrum originates instead from a monomer umbrella-like inversion motion within the complex. This assignment further supports the view that large amplitude vibrational motions complicate the correlation of a spectroscopically determined structure with the equilibrium structure.

Recent measurements of the dipole moment and quadrupole coupling constants in the $G$ states of the ammonia dimer have confirmed the nonrigid nature of the complex. ${ }^{9}$ A remarkable variation in the dipole moment with $K$ was found [ $\mu=0.74$ (2) $\mathrm{D}$ for $K=0$ and $\mu=0.10$ (1) D for $K=1]$. However, the departure of these small values from the large values expected for a hydrogen bonded structure is considered too great to arise from a vibrational averaging 
effect, and the measurements are viewed as support for the conclusion that $\left(\mathrm{NH}_{3}\right)_{2}$ is not a linearly hydrogen bonded system.

The spectrum and structure of the dimethylamine (DMA) dimer is interesting because DMA is closely related to ammonia. As for ammonia, it is commonly believed that DMA can act both as a hydrogen bond donor and as an acceptor. However, the hydrogen bonding interaction in (DMA $)_{2}$ might be expected to be stronger than in $\left(\mathrm{NH}_{3}\right)_{2}$ because both the donating and accepting properties of DMA are enhanced by an inductive effect from the two methyl groups. Specifically, it was determined using ion cyclotron resonance mass spectroscopy that DMA is both more acidic than ammonia (in the gas phase) ${ }^{10}$ and that its proton affinity is $16 \mathrm{kcal} / \mathrm{mol}$ greater than for ammonia. ${ }^{11}$ Thus simple primary and secondary amines may be a better model for the more complicated amines found in chemistry and biology.

Methyl- and dimethylamine complexes have received relatively little attention compared to the ammonia dimer. An electric deflection experiment determined that the dimers of DMA and methylamine have dipole moments greater than $0.3 \mathrm{D}^{12}$ This result was taken to support a linearly hydrogen bonded dimer structure over a cyclic, centrosymmetric structure. A linearly hydrogen bonded structure was also used to interpret the photoelectron spectrum of (DMA $)_{2} \cdot{ }^{13}$ The infrared photodissociation spectrum of the monomethylamine dimer contains a doublet feature in the $\mathrm{C}-\mathrm{N}$ stretch region, which suggested that the two nitrogens are in inequivalent positions within the dimer structure. ${ }^{14}$ Matrix Fourier transform infrared (FTIR) spectra have been recorded for (DMA) $)_{2}$ and other amine/ammonia complexes; ${ }^{15}$ the spectral shifts observed upon complexation were compared to those for the ammonia dimer and it was deduced that (DMA) ${ }_{2}$ and the other amine complexes have structures similar to the ammonia dimer.

This report presents the rotational spectra of four (DMA $)_{2}$ isotopomers; the structure of the dimer is discussed in terms of the moments of inertia, dipole moment, and nuclear quadrupole coupling constants. The results are also compared to the previous studies of the ammonia dimer.

\section{EXPERIMENT}

Rotational spectra for the dimethylamine complexes were recorded using a Balle-Flygare ${ }^{16}$ type Fouriertransform microwave spectrometer $(4-18 \mathrm{GHz})$. Two nozzle configurations were employed. With the gas expansion oriented perpendicular to the microwave cavity, the rotational linewidths [full width at half-maximum (FWHM)] are found to be approximately $30 \mathrm{kHz}$ (for transitions unsplit by nuclear quadrupole hyperfine structure) and the line centers were accurate to $\pm 4 \mathrm{kHz}$. When the expansion proceeds along the axis of the cavity, Doppler doublets separated by $50 \mathrm{kHz}$ and with linewidths of less than 15 $\mathrm{kHz}$ were observed; the axial nozzle configuration was used to resolve many of the closely spaced nuclear quadrupole hyperfine transitions. A modified Bosch fuel injector
TABLE I. Rotational transition frequencies of $\left(\mathrm{DMA}-{ }^{14} \mathrm{~N}\right)_{2}$ and $\left(\mathrm{DMA}-{ }^{15} \mathrm{~N}\right)_{2}$.

\begin{tabular}{|c|c|c|c|c|}
\hline $\begin{array}{l}\text { Transition } \\
J_{K_{P} K_{O}}^{\prime}-J_{K_{P} K_{O}}^{\prime \prime}\end{array}$ & $\begin{array}{c}\left(\mathrm{DMA}-{ }^{14} \mathrm{~N}\right)_{2}{ }^{\mathrm{a}} \\
(\mathrm{MHz})\end{array}$ & $\begin{array}{c}\text { Obs.-calc. } \\
(\mathrm{kHz})\end{array}$ & $\begin{array}{c}\left(\mathrm{DMA}-{ }^{15} \mathrm{~N}\right)_{2} \\
(\mathrm{MHz})\end{array}$ & $\begin{array}{c}\text { Obs.-calc. } \\
(\mathrm{kHz})\end{array}$ \\
\hline $3_{03}-2_{02}$ & & & 7721.846 & -0.6 \\
\hline $3_{12}-2_{11}$ & 8319.026 & -0.9 & 8208.731 & -0.5 \\
\hline $3_{22}-2_{21}$ & 7897.093 & 1.0 & 7798.421 & 1.8 \\
\hline $3_{21}-2_{20}$ & & & 7875.143 & 0.1 \\
\hline $4_{04}-3_{03}$ & 10330.419 & -0.8 & 10209.926 & 1.9 \\
\hline $4_{14}-3_{13}$ & 9910.968 & 0.1 & 9798.050 & -0.6 \\
\hline $4_{13}-3_{12}$ & 11065.785 & 1.6 & 10920.284 & 2.9 \\
\hline $4_{23}-3_{22}$ & 10513.384 & -0.7 & 10382.736 & 0.0 \\
\hline $4_{22}-3_{21}$ & & & 10570.753 & -1.8 \\
\hline $4_{32}-3_{31}$ & 10567.475 & 2.4 & 10433.792 & 0.9 \\
\hline $4_{31}-3_{30}$ & 10572.901 & -2.6 & 10438.757 & -3.5 \\
\hline $5_{05}-4_{04}$ & 12779.417 & -0.6 & 12635.449 & 1.9 \\
\hline $5_{15}-4_{14}$ & 12355.916 & 0.8 & 12216.483 & -1.1 \\
\hline $5_{14}-4_{13}$ & & & 13608.124 & -1.5 \\
\hline $6_{06}-5_{05}$ & & & 15001.676 & -1.3 \\
\hline $6_{16}-5_{15}$ & & & 14618.191 & 0.3 \\
\hline $2_{11}-1_{01}$ & 8772.652 & 1.6 & 8698.035 & 2.2 \\
\hline $220-2_{12}$ & 9673.647 & -0.5 & 9670.764 & 0.7 \\
\hline $22_{21}-2_{11}$ & & & 8805.962 & -2.3 \\
\hline $22_{20}-1_{10}$ & & & 14306.180 & -1.2 \\
\hline $2_{21}-1_{11}$ & & & 14568.676 & -0.3 \\
\hline $3_{21}-3_{13}$ & 10202.365 & 0.5 & 10181.779 & -1.4 \\
\hline $3_{12}-2_{02}$ & 11847.303 & -1.5 & 11727.005 & -0.2 \\
\hline $3_{22}-3_{12}$ & & & 8395.652 & -0.1 \\
\hline $3_{21}-2_{11}$ & & & 16700.437 & -1.2 \\
\hline $3_{22}-2_{12}$ & & & 17449.850 & -1.5 \\
\hline $4_{13}-3_{03}$ & & & 14925.440 & 0.3 \\
\hline $5_{23}-5_{15}$ & & & 12053.720 & 0.8 \\
\hline $4_{22}-4_{14}$ & & & 10954.488 & 3.4 \\
\hline $5_{05}-4_{13}$ & & & 7919.934 & 2.5 \\
\hline $4_{23}-4_{13}$ & & & 7858.110 & 3.1 \\
\hline
\end{tabular}

${ }^{a}$ Center frequencies obtained by fitting the nuclear quadrupole hyperfine transitions.

(model 0280150045 ) served as the pulsed valve. An electric field for Stark effect measurements was established by charging two $50 \mathrm{~cm} \times 50 \mathrm{~cm}$ steel mesh grids placed above and below the cavity and separated by $30 \mathrm{~cm}$. Up to $10000 \mathrm{~V}$ of opposite polarity can be applied to the grids; the electric field was calibrated by measuring the Stark shift of the $1 \leftarrow 0$ transition of OCS $[\mu=0.715196 \mathrm{D}$ (Ref. 17)].

The samples contained approximately $2 \%$ of either DMA (Linde) or DMA $-{ }^{15} \mathrm{~N}\left(99 \%{ }^{15} \mathrm{~N}\right.$, Isotech Inc.) in $1.6 \mathrm{~atm}$ of a 20:80 helium/neon carrier gas mix. The DMA $-{ }^{15} \mathrm{~N}$ was liberated from a hydrochloride salt by adding excess $\mathrm{NaOH}$ and collecting the evolved gas in a liquid nitrogen trap. The two (DMA $\left.-{ }^{15} \mathrm{~N}\right)-\left(\mathrm{DMA}-{ }^{15} \mathrm{~N}^{13} \mathrm{C}\right)$ isomers were observed in natural abundance using the DMA${ }^{15} \mathrm{~N}$ sample. The mixed $\left({ }^{15} \mathrm{~N},{ }^{14} \mathrm{~N}\right)$ DMA dimers were observed using a mixture containing $1 \%$ normal DMA and $1 \%$ DMA- $-{ }^{15}$.

\section{RESULTS}

\section{A. Microwave spectra}

Ten $a$ - and four $c$-type rotational transitions were measured for the DMA $-{ }^{14} \mathrm{~N}$ dimer (Table I); each rotational 
TABLE II. Rotational constants, second moments, centrifugal distortion constants, and quadrupole coupling constants for the (DMA) 2 isotopomers.

\begin{tabular}{|c|c|c|c|c|c|c|}
\hline & $\left(\mathrm{DMA}-{ }^{14} \mathrm{~N}\right)_{2}$ & $\left(\mathrm{DMA}-{ }^{15} \mathrm{~N}\right)_{2}$ & $\begin{array}{l}\left(\mathrm{DMA}-{ }^{14} \mathrm{~N}\right)- \\
\left(\mathrm{DMA}-{ }^{15} \mathrm{~N}\right)\end{array}$ & $\begin{array}{l}\left(\mathrm{DMA}-{ }^{15} \mathrm{~N}\right)- \\
\left(\mathrm{DMA}-{ }^{14} \mathrm{~N}\right)\end{array}$ & $\begin{array}{c}\left(\mathrm{DMA}-{ }^{15} \mathrm{~N}^{13} \mathrm{C}\right)- \\
\left(\mathrm{DMA}-{ }^{15} \mathrm{~N}\right)\end{array}$ & $\begin{array}{l}\left(\mathrm{DMA}-{ }^{15} \mathrm{~N}\right)- \\
\left(\mathrm{DMA}-{ }^{15} \mathrm{~N}^{13} \mathrm{C}\right)\end{array}$ \\
\hline$A(\mathrm{MHz})$ & $4388.908(3)$ & $4376.022(2)$ & $4386.892(1)$ & $4378.066(1)$ & $4320.554(1)$ & $4323.022(2)$ \\
\hline$B(\mathrm{MHz})$ & $1461.273(1)$ & $1440.695(1)$ & $1453.508(1)$ & $1448.540(1)$ & $1425.504(1)$ & $1422.605(1)$ \\
\hline$C(\mathrm{MHz})$ & $1171.182(1)$ & $1158.867(1)$ & $1166.347(1)$ & $1163.780(1)$ & $1145.440(1)$ & $1143.459(1)$ \\
\hline$D_{J}(\mathrm{kHz})$ & $0.98(1)$ & $0.973(4)$ & $0.992(2)$ & $0.978(3)$ & $0.959(4)$ & $0.944(6)$ \\
\hline$D_{J K}(\mathrm{kHz})$ & $7.16(3)$ & $6.94(2)$ & $7.04(8)$ & $6.8(1)$ & $6.7(1)$ & $6.8(2)$ \\
\hline$D_{k}(\mathrm{kHz})$ & $-6.9(6)$ & $-6.6(4)$ & & & & \\
\hline$d_{1}(\mathrm{kHz})$ & $-0.23(1)$ & $-0.218(3)$ & $-0.213(3)$ & $-0.205(4)$ & $-0.200(5)$ & $-0.216(6)$ \\
\hline$d_{2}(\mathrm{kHz})$ & $-0.053(9)$ & $-0.054(3)$ & & & & \\
\hline$P_{o a}\left(\mathrm{amu} \AA^{2}\right)$ & $331.1056(4)$ & $335.6988(4)$ & $332.8974(4)$ & $333.8554(4)$ & $339.3826(4)$ & $340.1594(4)$ \\
\hline$P_{b b}\left(\mathrm{amu} \AA^{2}\right)$ & $100.4063(1)$ & $100.3987(1)$ & $100.4034(1)$ & $100.4011(1)$ & $101.8269(1)$ & $101.8145(1)$ \\
\hline$P_{\kappa}\left(\operatorname{amu} \AA^{2}\right)$ & $14.74284(2)$ & $15.08953(2)$ & $14.79870(2)$ & $15.03318(2)$ & $15.14396(2)$ & $15.08962(2)$ \\
\hline$\chi_{a d}(1)(\mathrm{MHz})$ & $-4.531(2)$ & & $-4.54(1)$ & & & \\
\hline$\chi_{b b}(1)(\mathrm{MHz})$ & $2.847(2)$ & & $2.86(1)$ & & & \\
\hline$\chi_{c r}(1)(\mathrm{MHz})$ & $1.684(3)$ & & $1.68(1)$ & & & \\
\hline$\chi_{\infty \infty}(2)(\mathrm{MHz})$ & $1.644(2)$ & & & $1.67(2)$ & & \\
\hline$\chi_{\Delta b}(2)(\mathrm{MHz})$ & $3.157(3)$ & & & $3.13(1)$ & & \\
\hline$\chi_{\propto c}(2)(\mathrm{MHz})$ & $-4.801(4)$ & & & $-4.80(2)$ & & \\
\hline
\end{tabular}

transition was split extensively into nuclear quadrupole hyperfine components arising from the two $I=1,{ }^{14} \mathrm{~N}$ nuclei. $\chi_{a a}(1), \chi_{b b}(1), \chi_{a a}(2), \chi_{b b}(2)$ (Table II), and the center frequencies of the rotational transitions were obtained by fitting the assigned nuclear quadrupole hyperfine components (available as supplemental material). ${ }^{18}\left(v_{\text {obs }}\right.$ $\left.-v_{\text {calc }}\right)_{\text {rms }}=3.4 \mathrm{kHz}$ for the 180 hyperfine components. The center frequencies were fit to the Watson $S$-reduction Hamiltonian using the $I^{r}$ representation and five quartic distortion constants $-\left(v_{\text {obs }}-v_{\text {calc }}\right)_{\text {rms }}=1.3 \mathrm{kHz}$. The rotational and distortion constants are listed in Table II. No tunneling splittings were found in the $2 \mathrm{GHz}$ region covered in the initial search to locate and assign the spectrum.

Thirty-one rotational transitions (Table I) were found for the DMA $-{ }^{15} \mathrm{~N}$ dimer. Again, three rotational and five quartic distortion constants (Table II) were calculated by fitting to the Watson Hamiltonian. $\left(v_{\mathrm{obs}}-v_{\mathrm{calc}}\right)_{\mathrm{rms}}=1.7$ $\mathrm{kHz}$ for the fit. The rotational transitions were very intense because of the absence of nuclear quadrupole splitting. For this reason, a search for tunneling doublets was undertaken over a $225 \mathrm{MHz}$ range centered at $10200 \mathrm{MHz}$. No additional transitions were found which could be assigned as tunneling doublets of either the $4_{04}-3_{03}$ or $3_{21}-3_{13}$ transitions. The intensity of the $\mathrm{DMA}-{ }^{15} \mathrm{~N}$ dimer rotational transitions also enabled us to locate and assign the spectra of two ${ }^{13} \mathrm{C}^{15} \mathrm{~N}_{2}$ isotopomers using the DMA- ${ }^{15} \mathrm{~N}$ sample. Ten rotational transitions were found for each of the ${ }^{13} \mathrm{C}$ isotopomers (available as supplemental material ${ }^{18}$ ) and their spectra were separated by approximately $15 \mathrm{MHz}$. Only the constants $A, B, C, D_{J}, D_{J K}$, and $d_{1}$ (Table II) were determined from the spectral fit because fewer transitions were measured. $\left(v_{\text {obs }}-v_{\text {calc }}\right)_{\text {rms }}<1 \mathrm{kHz}$ for both fits. Nine rotational transitions were found for each of the two (DMA- $\left.{ }^{14} \mathrm{~N}\right)-\left(\mathrm{DMA}-{ }^{15} \mathrm{~N}\right)$ dimers. The nuclear quadrupole hyperfine transitions (available as supplemental material $^{18}$ ) were fit to yield center frequencies and quadrupole coupling constants. Again, $A, B, C, D_{J}, D_{J K}$, and $d_{1}$ (Ta- ble II) were obtained from the spectral fit; $\left(v_{\text {obs }}-v_{\text {calc }}\right)<1$ $\mathrm{kHz}$ for both fits.

Measurements of Stark shifts were made for the $3_{03}-$ 202 and $4_{04}-3_{03}$ transitions of the DMA $-{ }^{15} \mathrm{~N}$ dimer to avoid the complicating effects of the nuclear quadrupole splitting. The observed Stark effects of six $|M|$ components (the Stark shift of the $3_{03}-2_{02}|M|=1$ component was too slow to be measured accurately) were initially fit to the $a$, $b$, and $c$ components of the dipole moment using second order Stark coefficients calculated from the rotational constants. The resulting values were $\mu_{a}^{2}=2.26 \mathrm{D}^{2}, \mu_{b}^{2}=-0.03$ $\mathrm{D}^{2}$, and $\mu_{c}^{2}=0.74 \mathrm{D}^{2}$; the small negative value for $\mu_{b}^{2}$ is consistent with $\mu_{b}=0.0(2) \mathrm{D}$. A second fit, with $\mu_{b}$ fixed at zero, yielded $\mu_{a}=1.50$ (1) D and $\mu_{c}=0.84(1) \mathrm{D}$. The observed and calculated Stark shifts are compared in Table III.

\section{B. Structure}

The spectroscopic data can provide structural information if the effects of vibrational averaging from the van der Waals modes and tunneling processes can be neglected.

TABLE III. A comparison of the observed and calculated ${ }^{a}$ Stark effects for (DMA- $\left.{ }^{15} \mathrm{~N}\right)_{2}$.

\begin{tabular}{cccc}
\hline \hline Transition & $|M|$ & $\begin{array}{c}\left(\Delta v / \epsilon^{2}\right) \\
{\left[\mathrm{MHz} /(\mathrm{kV} / \mathrm{cm})^{2}\right]} \\
\text { observed }\end{array}$ & $\begin{array}{c}\left(\Delta v / \epsilon^{2}\right) \\
{\left[\mathrm{MHz} /(\mathrm{kV} / \mathrm{cm})^{2}\right]} \\
\text { calculated }^{\mathrm{a}}\end{array}$ \\
\hline $3_{03}-2_{02}$ & 0 & $-7.27(1)$ & -7.29 \\
& 2 & $21.30(1)$ & 21.29 \\
$4_{04}-3_{03}$ & 0 & $-2.63(1)$ & -2.63 \\
& 1 & $-0.92(1)$ & -0.91 \\
& 2 & $4.26(1)$ & 4.27 \\
& 3 & $12.89(1)$ & 12.90 \\
\hline
\end{tabular}

${ }^{a}$ Calculated values using $\mu_{a}=1.50 \mathrm{D}, \mu_{b}=0 \mathrm{D}, \mu_{c}=0.84 \mathrm{D}$, and the rotational constants in Table II. 


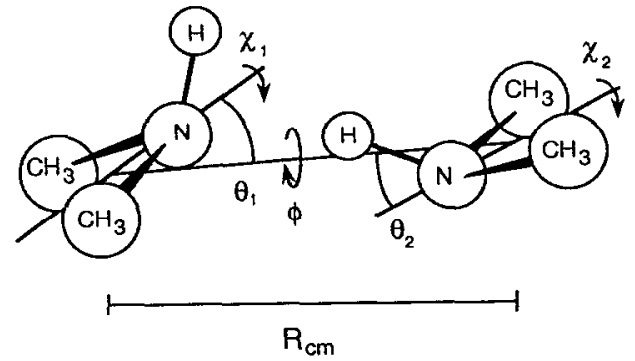

FIG. 1. Geometrical parameters used to describe the structure of the dimethylamine dimer, $R_{c . m}$ is the distance between the monomer centers of mass, $\theta_{1}$ and $\theta_{2}$ are the angles between the $\boldsymbol{R}_{\mathrm{c} . \mathrm{m} \text {. vectors and lines }}$ between centers of mass and the nitrogen atoms for the two monomers, $\chi_{1}$ and $\chi_{2}$ are torsional angles about lines between the centers of mass and the nitrogen atoms for the two monomers, and $\phi$ is the dihedral angle between the symmetry planes of the two monomer units.

This approximation seems reasonable (see the discussion) at the outset. The relatively small centrifugal distortion constants and the absence of tunneling splittings suggest that the vibrational motions are similar to other complexes where moments of inertia uncorrected for vibrations can be used to determine structural parameters.

Two observations indicate that the DMA dimer has a plane of symmetry containing the $a$ and $c$ inertial axes. First, $P_{b b}$ is the same for $\left(\mathrm{DMA}-{ }^{14} \mathrm{~N}\right)_{2},\left(\mathrm{DMA}-{ }^{15} \mathrm{~N}\right)_{2}$, and the two $\left(\mathrm{DMA}-{ }^{14} \mathrm{~N}\right)-\left(\mathrm{DMA}-{ }^{15} \mathrm{~N}\right)$ complexes (Table II), so both nitrogen atoms must lie in the $a c$ plane. Second, $\mu_{b}$ of the dimer was determined to be zero by fitting the observed Stark shifts, which is consistent with an $a c$ symmetry plane. Since the DMA monomer has a plane of symmetry which contains the nitrogen atom and the dipole moment, the monomer symmetry planes must be coplanar with the dimer $a c$ plane. The observed $P_{b b}$ (100.41 amu $\AA^{2}$ ) compares well with the value expected from the DMA monomer ${ }^{19}$ (100.90 amu $\AA^{2}$ ).

The structure of the DMA dimer can be determined more quantitatively by fitting the dimer structure to the moment-of-inertia data for the six isotopomers. Assuming that the monomer structure ${ }^{19}$ is unchanged upon complexation, six parameters are required to describe the complex structure. These six parameters are shown in Fig. 1 and are labeled in the same manner as for the ammonia dimer. ${ }^{1}$ As usual, $R_{\text {c.m. }}$ is the center-of-mass (c.m.) separation; $\theta_{1}$ and $\theta_{2}$ are angles between the $R_{\text {c.m. }}$. vector and a line joining the c.m. to the nitrogen atom for molecules 1 and 2. $\chi_{1}$ and $\chi_{2}$ are torsional angles about the two c.m.-nitrogen lines and $\phi$ represents a torsional angle about the $R_{\text {c.m. }}$ vector. Although defined in a similar manner as for the ammonia dimer, the angular and torsional parameters are not exactly the same. The difference arises because the ammonia c.m. lies on a $C_{3}$ axis-equidistant from the three protons. In dimethylamine, the c.m. lies in a mirror plane, but much closer to the methyl groups than the nitrogen proton. This difference indicates that $\theta_{1}$ and $\theta_{2}$ for DMA dimers cannot be compared directly to the experimental values for the ammonia dimer (see the discussion for a comparison to the
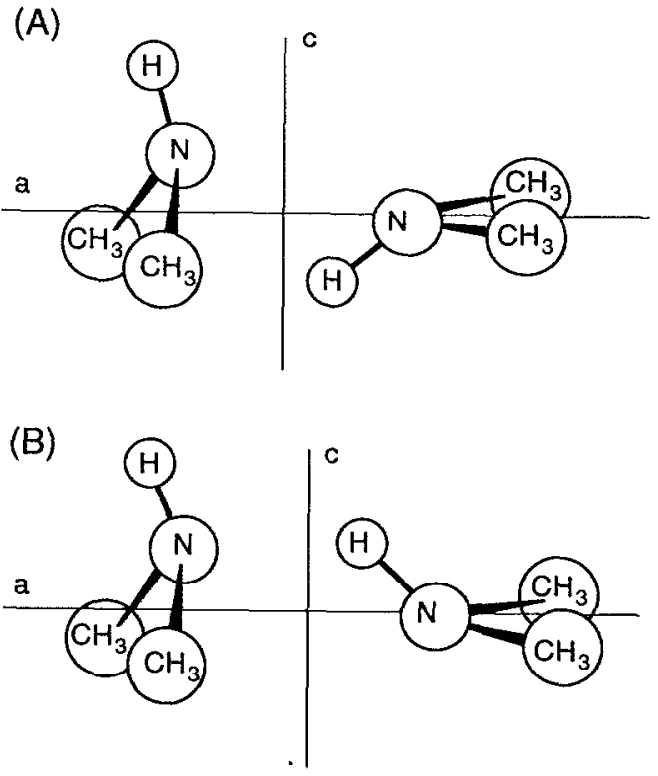

FIG. 2. Two dimethylamine dimer structures (in their principal axes systems) which best fit the moment-of-inertia data. (B) is preferred on the basis of the electrostatic calculations (see the text).

ammonia dimer; see also the Appendix for some additional details on the structural fitting).

Because the DMA dimer was shown above to possess a plane of symmetry, $\chi_{1}, \chi_{2}$, and $\phi$ can be fixed at either $0^{\circ}$ or $180^{\circ} . \theta_{1}, \theta_{2}$, and $R_{\text {c.m. }}$. were fit to best match the moments of inertia of the six isotopomers. Two structures were found that model the data equally well; these structures are shown in Figs. 2(A) $\left(\phi=0^{\circ}\right)$ and 2(B) $(\phi$ $\left.=180^{\circ}\right)$. The remaining parameters were equal for the two structures $\theta_{1}=69^{\circ}(7), \theta_{2}=1^{\circ}(10), R_{\text {c.m. }}=3.746(1) \AA$, and $\Delta I_{\mathrm{rms}}=0.331 \mathrm{amu} \AA^{2}\left[\Delta I=I_{x}(\mathrm{obs})-I_{x}(\mathrm{calc}) ; x=a, b\right.$, or $c]$. Neither structure contains a linear hydrogen bond. Distinguishing between structures $2(\mathrm{~A})$ and $2(\mathrm{~B})$ using only moments of inertia is difficult. The centers of mass of the two monomers lie very close to the $a$ axis, so a change of $\phi$ by $180^{\circ}$ is nearly equivalent to a rotation of one monomer about the $a$ axis by $180^{\circ}$-an operation which does not change the moments of inertia (because of the monomer plane of symmetry). Thus structures 2(A) and 2(B) will always have very similar moments of inertia and the spectra of isotopically substituted dimers will not distinguish between the two structures.

Two linearly hydrogen bonded dimers were also considered $\left\{\left[\right.\right.$ Fig. 3(A)] $\theta_{1}=42.0^{\circ}, \theta_{2}=39.5^{\circ}$, and $\phi=0^{\circ}$; [Fig. 3(B)] $\theta_{1}=\theta_{2}=56.2^{\circ}$ and $\left.\phi=180^{\circ}\right\}$. Only $R_{\text {c.m. }}$ was fit to the moment-of-inertia data, yielding $R_{\text {c.m. }}=3.744(2) \AA$ and $\Delta I_{\mathrm{rms}}=0.887 \mathrm{amu} \AA^{2}$ for structure 3(A), and $R_{\text {c.m. }}$. $=3.752(4) \AA$ and $\Delta I_{\text {rms }}=2.082$ amu $\AA^{2}$ for structure 3 (B). Neither linearly hydrogen bonded structure fits the moment-of-inertia data as well as structures $2(\mathrm{~A})$ and $2(B)$. The fit for structure $3(B)$ is poor enough to readily eliminate it. The fit for structure 3(A), while less egregious, is also troublesome. For example, structure 3(A) does not fit the nitrogen or carbon isotopic shift data as 
TABLE IV. A comparison of carbon and nitrogen atomic positions in (Ångstroms) calculated by various methods. ${ }^{2}$

\begin{tabular}{|c|c|c|c|c|c|c|c|c|}
\hline & & & \multicolumn{6}{|c|}{ Least squares fitting ${ }^{c}$} \\
\hline & \multicolumn{2}{|c|}{ Kraitchman $^{\mathrm{b}}$} & \multicolumn{2}{|c|}{ Structure $2(\mathrm{~A})$} & \multicolumn{2}{|c|}{ Structure 2(B) } & \multicolumn{2}{|c|}{ Structure 3(A) } \\
\hline & $\mathrm{C}_{L}$ & $\mathrm{C}_{R}$ & $\mathrm{C}_{L}$ & $\mathrm{C}_{R}$ & $\mathrm{C}_{L}$ & $\mathrm{C}_{R}$ & $\mathrm{C}_{L}$ & $\mathrm{C}_{R}$ \\
\hline$|a|(\AA)$ & 1.926 & 2.120 & 1.942 & 2.131 & 1.934 & 2.132 & 2.058 & 2.079 \\
\hline$|b|(\AA)$ & 1.193 & 1.188 & 1.214 & 1.214 & 1.214 & 1.214 & 1.214 & 1.214 \\
\hline \multirow[t]{2}{*}{$|c|(\AA)$} & 0.236 & 0.009 & 0.240 & 0.009 & 0.258 & 0.028 & 0.142 & 0.116 \\
\hline & $\mathbf{N}_{L}$ & $\mathbf{N}_{R}$ & $\mathbf{N}_{L}$ & $\mathbf{N}_{R}$ & $\mathbf{N}_{L}$ & $\mathbf{N}_{R}$ & $\mathbf{N}_{L}$ & $\mathbf{N}_{R}$ \\
\hline$|\alpha|(\AA)$ & 1.668 & 1.352 & 1.663 & 1.317 & 1.679 & 1.317 & 1.433 & 1.448 \\
\hline$|b|(\AA)$ & 0.000 & 0.000 & 0.000 & 0.000 & 0.000 & 0.000 & 0.000 & 0.000 \\
\hline$|c|(\AA)$ & 0.535 & 0.236 & 0.526 & 0.022 & 0.516 & 0.008 & 0.383 & 0.402 \\
\hline
\end{tabular}

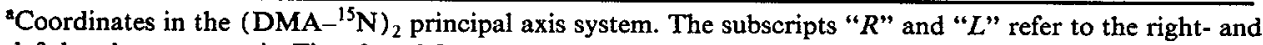
left-hand monomers in Figs. 2 and 3.

bCalculated using Kraitchman's single substitution equations for isotopic shifts (Ref. 20).

'From least squares fitting the moments of inertia of the six isotopic species.

satisfactorily as structures $2(\mathrm{~A})$ and $2(\mathrm{~B})$. This is apparent by comparing the carbon and nitrogen coordinates calculated from Kraitchman's single isotopic substitution equations ${ }^{20}$ with those obtained for the three structures obtained by least squares fitting the moments (Table IV). The $\Delta I_{\text {rms }}$ for structure $3(\mathrm{~A})$ also seems to be too large to be acceptable. In studies over the last five years of some 20 systems in our laboratory, we have found that complexes involving heavy monomers and with no unusual tunneling or large amplitude effects usually give least squares fits with $\Delta I_{\text {rms }} \leqslant 0.5 \mathrm{amu} \AA^{2}$. In summary, the isotopic data support the cyclic structures $2(\mathrm{~A})$ and $2(\mathrm{~B})$ over conformers with linear hydrogen bonds, such as structures $3(A)$ and $3(B)$.
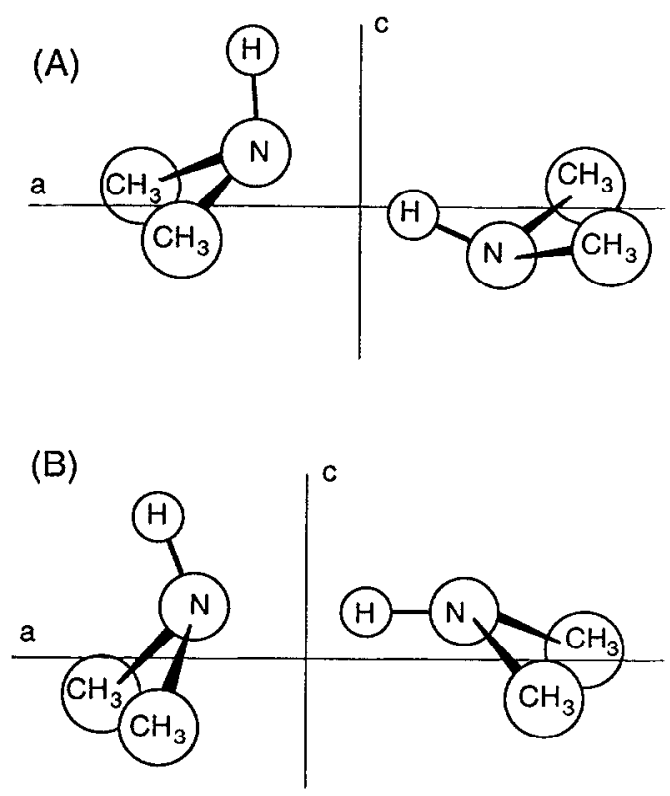

FIG. 3. Diagrams of two linearly hydrogen bonded complexes. In (A), the hydrogen of the left-hand monomer is staggered with respect to the two methyl groups of the right-hand monomer. In (B), the methyl groups are eclipsed with respect to each other.
Structural information can often be discerned from the dipole moment components. However, in this case, polarization effects seem large and obscure the interpretation. The dipole components expected for structures 2(A), 2(B), and 3(A) based on the dipole moment of DMA are compared in Table V. For typical van der Waals complexes, the induced dipole moment is largest along the $a$ axis and this component is on the order of $0.2-1.0 \mathrm{D}$. On the other hand, the induced moments along the $b$ and $c$ axes are usually small $(0.05-0.2 \mathrm{D})$ and often antiparallel (i.e., they reduce the observed value below the value predicted from the monomers without polarization). These general trends seem to support structure $2(B)$. However, this is far from conclusive since the structure of (DMA) is somewhat unusual and it is not clear that the usual polarization trends should be followed in this case.

Structural information can also be obtained from the nuclear quadrupole coupling constants by rotating the quadrupole coupling tensor of the monomer into the dimer principal axis system. The rotation depends only on the magnitudes of the angles between the monomer and dimer principal axis systems [which are nearly the same for structures 2(A) and 2(B)], so again, the experimental data cannot distinguish between structures $2(\mathrm{~A})$ and $2(\mathrm{~B})$. Since the angles between the quadrupole principal axes and inertial principal axes are known for the monomer, ${ }^{19}$ it is possible to calculate similar angles for the three dimer structures. In both structures $2(\mathrm{~A})$ and $2(\mathrm{~B})$, the angles between the two monomer $z$ principal quadrupole axes and the dimer $a$ inertial axis are $14^{\circ}$ and $59^{\circ}$, while in structure

TABLE V. A comparison of dipole components $(D)$ measured for $\left(D M A-{ }^{15} \mathrm{~N}\right)_{2}$ with dipole components calculated for various structures.

\begin{tabular}{ccccc}
\hline \hline & Obs. & Structure 2(A) & Structure 2(B) & Structure 3(A) \\
\hline$\mu_{a}$ & 1.50 & 0.59 & 0.59 & 1.16 \\
$\mu_{c}$ & 0.84 & 1.06 & 0.78 & 1.40 \\
\hline \hline
\end{tabular}


$3(\mathrm{~A})$, the angles are $19^{\circ}$ and $82^{\circ}$. The two sets of dimer quadrupole coupling constants determined spectroscopically are reproduced if the two monomer $z$ quadrupole axes make angles of $14^{\circ}$ and $77^{\circ}$ with the $a$ inertial axis of the dimer. These values are close for all three structures and cannot distinguish among them because electronic effects and vibrational averaging have been neglected. It is interesting that the out-of-plane coupling constant in the DMA monomer is $3.02 \pm 0.2 \mathrm{MHz}$, which straddles the values for $\chi_{b b}$ found in (DMA) ${ }_{2}$ (Table II). This similarity suggests that the vibrational and electronic effects on the coupling constants are not particularly large or unusual.

\section{DISCUSSION}

The DMA dimer exhibits no unusually large amplitude vibrational effects in its rotational spectrum and derived constants. There is no reason to suspect that a least squares fitting of the moments of inertia should not result in a good approximation of the equilibrium conformation. These inertial data are most compatible with structures 2(A) and 2(B). Forcing the inertial data to model linearly hydrogen bonded forms $3(A)$ and $3(B)$ seriously degrades the overall inertial fit quality. In the absence of any other strong evidence for the latter two structures, they will no longer be considered.

Selecting between structures $2(A)$ and $2(B)$ on the basis of experimental evidence is difficult, as mentioned above. Chemical intuition, however, immediately suggests that structure $2(\mathrm{~A})$ is less likely because the two nitrogen lone pairs are directed toward each other. To quantify intuition, two computational approaches were used to determine the relative stabilities of structures $2(\mathrm{~A})$ and $2(\mathrm{~B})$. A complete theoretical treatment of the DMA dimer, however, is beyond the scope of this report, since very sophisticated techniques have been used to model the ammonia dimer. For this reason, we have limited our calculations to structures $2(\mathrm{~A})$ and $2(\mathrm{~B})$. Gaussian $90^{21}$ was used the Hartree-Fock level with the $6-31 \mathrm{G}^{* *}$ basis set to calculate the relative energies of the DMA monomer and structures 2(A) and 2(B). The energies were found to be -134.25062 a.u. for the monomer, -268.49167 a.u. for structure $2(\mathrm{~A})$, and -268.50124 a.u. for structure $2(\mathrm{~B})$. The binding energies, therefore, are +0.00957 a.u. (+6.01 $\mathrm{kcal} / \mathrm{mol}$, unstable) for structure $2(\mathrm{~A})$ and -0.00126 a.u. ( $-0.792 \mathrm{kcal} / \mathrm{mol}$, stable) for structure 2(B). A semiempirical electrostatic calculation of the structural stabilities was undertaken using the model of Buckingham and Fowler ${ }^{22}$ and distributed multipoles (available as supplementary material ${ }^{18}$ ) calculated using the Cambridge Analytic Derivatives Package (CADPAC) routine $^{23}$ with the $6-31 \mathrm{G}^{* *}$ basis set. The energies were found to be $+4.731 \mathrm{kcal} / \mathrm{mol}$ for structure $2(\mathrm{~A})$ and $-1.729 \mathrm{kcal} / \mathrm{mol}$ for structure $2(\mathrm{~B})$. The $a b$ initio and electrostatic calculations confirm chemical intuition and indicate that structure $2(\mathrm{~A})$ is unstable. The cyclic structure $2(B)$ is, therefore, preferred.

It is interesting to compare structure $2(B)$ with the earlier cyclic structure proposed by the Klemperer group for the ammonia dimer. ${ }^{1}$ The comparison requires some additional geometric manipulation since the fitted values of $\theta_{1}$ and $\theta_{2}$ cannot be compared to the corresponding values for the ammonia dimer (as discussed above). To make a more direct comparison, we defined a "pseudo- $C_{3}$ " axis such that the $\mathrm{N}-\mathrm{H}$ and $\mathrm{N}-\mathrm{C}$ bonds make the same angle $\left(109^{\circ}\right)$ with this axis. We then calculated the values of the angles $\left(\alpha_{1}\right.$ and $\left.\alpha_{2}\right)$ between the pseudo- $C_{3}$ axes and a line joining the two nitrogens. For the ammonia dimer, $\theta_{1}$ and $\theta_{2}$ are defined with respect to a line joining the monomer centers of mass, but since the ammonia center of mass is less than $0.1 \AA$ from the nitrogen atom, the angles $\alpha$ should be comparable to the ammonia dimer $\theta$ angles. We obtained $\alpha_{1}=29^{\circ}$ and $\alpha_{2}=65^{\circ}$ for structure 2(B). Analysis of the ammonia dimer dipole and quadrupole coupling constants ${ }^{1}$ yielded $\theta_{1}=48.6^{\circ}$ and $\theta_{2}=64.5^{\circ}$, so the two cyclic structures are similar. Given the highly nonrigid nature of $\left(\mathrm{NH}_{3}\right)_{2}$, it is unclear whether the similarity is fortuitous and whether a quantitative comparison of these angles is really meaningful at this point. Nevertheless, because (DMA $)_{2}$ is more likely to form a linearly hydrogen bonded complex than ammonia (based on the acidity/ basicity arguments given in the Introduction), the evidence for a cyclic DMA dimer appears significant. This kind of interaction is noteworthy and may be more generally found in amines than previously considered.

\section{ACKNOWLEDGMENTS}

This work was supported by a grant from the National Science Foundation. We thank Fu-Ming Tao and W. Klemperer for a preprint of their work.

\section{APPENDIX: STRUCTURAL FITTING}

The least squares fitting proceeded by starting with trial structures with $C_{s}$ symmetry, with the amine hydrogens either trans [Fig. 2(A)] or cis [Fig. 2(B)] to each other and using the rotational constants from (DMA$\left.{ }^{14} \mathrm{~N}\right)_{2}$, (DMA- $\left.{ }^{15} \mathrm{~N}\right)_{2}$, and the two ${ }^{13} \mathrm{C}$ isotopomers. The two sets of ${ }^{13} \mathrm{C}$ isotopomer rotational constants were assigned to the left-hand carbon (of Fig. 2, $\mathrm{C}_{L}$ )/right-hand carbon $\left(C_{R}\right)$ or vice versa. The trans structure converged to the trans cyclic isomer; the cis converged to the cis cyclic isomer. Exchanging the assignment of the ${ }^{13} \mathrm{C}$ isotopomers did not change the fit, since the same two structures were found. The rotational constants of the mixed (DMA$\left.{ }^{14} \mathrm{~N}\right)-\left(\mathrm{DMA}-{ }^{15} \mathrm{~N}\right)$ isotopomers were predicted by these fits; these predictions were used as a guide in correctly assigning the two $\left({ }^{15} \mathrm{~N},{ }^{14} \mathrm{~N}\right)$ mixed isotopomers, which were then included in the final fits of the cis and trans structures. The coordinates of all the atoms in the least squares fit structures are available as supplementary material. ${ }^{18}$ The analysis for the linearly hydrogen bonded structures in Fig. 3 proceeded analogously, except that $\theta_{1}$ and $\theta_{2}$ were constrained. The final fit coordinates for these structures are also provided as supplementary material. ${ }^{18}$

${ }^{1}$ D. D. Nelson, Jr., G. T. Fraser, and W. Klemperer, J. Chem. Phys. 83, 6201 (1985); D. D. Nelson, Jr., W. Klemperer, G. T. Fraser, F. J. Lovas, and R. D. Suenram, ibid. 87, 6364 (1987). 
${ }^{2}$ D. D. Nelson, Jr., G. T. Fraser, and W. Klemperer, Science 238, 1670 (1987).

${ }^{3}$ K. P. Sagarik, R. Ahlrichs, and S. Brode, Mol. Phys. 57, 1247 (1986); Z. Latajka and S. Scheiner, J. Chem. Phys. 84, 341 (1986).

${ }^{4}$ F.-M. Tao and W. Klemperer, J. Chem. Phys. 99, 5976 (1993).

${ }^{5}$ (a) M. J. Frisch, J. A. Pople, and J. E. Del Bene, J. Phys. Chem. 89, 3664 (1985); (b) M. J. Frisch, J. E. Del Bene, J. S. Binkley, and H. F. Schaefer III, J. Chem. Phys. 84, 2279 (1986); (c) S.-Y. Liu, C. E Dykstra, K. Kolenbrander, and J. M. Lisy, ibid. 85, 2077 (1986); (d) D. M. Hassett, C. J. Marsden, and B. J. Smith, Chem. Phys. Lett. 183, 449 (1991).

'J. W. I. van Bladel, A. van der Avoird, P. E. S. Wormer, and R. J. Saykally, J. Chem. Phys. 97, 4750 (1992).

${ }^{7}$ M. Havenith, R. C. Cohen, K. L. Busarow, D.-H. Gwo, Y. T. Lee, and R. J. Saykally, J. Chem. Phys. 94, 4776 (1991).

${ }^{8}$ J. G. Loeser, C. A. Schmuttenmaer, R. C. Cohen, M. J. Elrod, D. W. Steyert, R. J. Saykally, R. E. Bumgarner, and G. A. Blake, J. Chem. Phys. 97, 4727 (1992).

${ }^{9}$ H. Linnartz, A. Kips, W. L. Meerts, and M. Havenith, J. Chem. Phys. 99, 2449 (1993).

${ }^{10}$ J. I. Brauman and L. K. Blair, J. Am. Chem. Soc. 93, 3911 (1971).

"D. H. Aue, H. M. Webb, and M. T. Bowers, J. Am. Chem. Soc. 98, 311 (1976).

12 J. A. Odutola, R. Viswanathan, and T. R. Dyke, J. Am. Chem. Soc. 101, 4787 (1979).

${ }^{13}$ T. Pradeep, M. S. Hegde, and C. N. R. Rao, J. Mol. Spectrosc. 150, 289 (1991).
${ }^{14}$ U. Buck, X. Gu, R. Krohne, C. Lauenstein, H. Linnartz, and A. Rudolf, J. Chem. Phys. 94, 23 (1991).

${ }^{15}$ R. B. Bohn and L. Andrews, J. Phys. Chem. 95, 9707 (1991).

${ }^{16}$ T. J. Balle and W. H. Flygare, Rev. Sci. Instrum. 52, 33 (1981); K. W. Hillig II, J. Matos, A. Scioly, and R. L. Kuczkowski, Chem. Phys. Lctt. 133, 359 (1987).

${ }^{17}$ K. Tanaka, H. Ito, K. Harada, and T. Tanaka, J. Chem. Phys. 80, 5893 (1984).

${ }^{18}$ See AIP document no. PAPS JCPSA-100-3377-13 for 13 pages of tables of dimethylamine dimer isotopic and structural data. Order by PAPS number and journal reference from American Institute of Physics, Physics Auxiliary Publication Service, 500 Sunnyside Boulevard, Woodbury, New York 11797-2999. The price is $\$ 1.50$ for each microfiche (60 pages) or $\$ 5.00$ for photocopies of up to 30 pages, and $\$ 0.15$ for each additional page over 30 pages. Air mail additional. Make checks payable to the American Institute of Physics.

${ }^{19}$ J. E. Wollrab and V. W. Laurie, J. Chem. Phys. 48, 5058 (1968).

${ }^{20}$ W. Gordy and R. L. Cook, Microwave Molecular Spectra (Wiley, New York, 1984), pp. 656-667.

${ }^{21}$ M. J. Frisch, M. Head-Gordon, G. W. Trucks, J. B. Foresman, H. B. Schlegel, K. Raghavachari, M. Robb, J. S. Binkley, C. Gonzalez, D. J. Defrees, D. J. Fox, R. A. Whiteside, R. Seeger, C. F. Melius, J. Baker, R. L. Martin, L. R. Kahn, J. J. P. Stewart, S. Topiol, and J. A. Pople, Gaussian 90, revision I, Gaussian, Inc., Pittsburgh, 1990.

${ }^{22}$ A. D. Buckingham and P. W. Fowler, J. Chem. Phys. 79, 6426 (1983); Can. J. Chem. 63, 2018 (1985).

${ }^{23}$ R. D. Amos and J. E. Rice, The Cambridge Analytic Derivatives Package, Issue 4.0, Cambridge, England, 1987. 